Research Article

\title{
User's Attitude under the Perspective of Mental Energy Flow
}

\author{
Jing Yang $\mathbb{D}$, Qingchao Zhao $\mathbb{D}$, Xin Zhao, Di Wu $\mathbb{D}$, Ming Li, and Wei Zhang $\mathbb{D}$ \\ College of Computer Science and Technology, Harbin Engineering University, Harbin, China \\ Correspondence should be addressed to Qingchao Zhao; zhaoqc418@hrbeu.edu.cn
}

Received 1 June 2019; Revised 21 January 2020; Accepted 12 February 2020; Published 11 March 2020

Academic Editor: Giuseppe D’Aniello

Copyright (c) 2020 Jing Yang et al. This is an open access article distributed under the Creative Commons Attribution License, which permits unrestricted use, distribution, and reproduction in any medium, provided the original work is properly cited.

\begin{abstract}
The analysis of the attitudes of online users plays an important role in real life and the future society, which can help us to analyze and predict the behavior of the users. The researchers also carried out a lot of related work. However, most of the previous classic methods searched the rules of the structure of the graph or the propagation path and did not consider the internal psychological dynamic mechanism of the user. In the context of big data, identifying online behavioral motivations of users through online information should be a future development trend. In this paper, we delve into the users' internal psychological energy based on the Elaboration Likelihood Model and divide the user's behavioral drive into the central route and the peripheral route. The degree of user's processing of the known information and elaboration determines which route is more effective. The central route is mainly the user's behavioral habits studied through user role theory. In the peripheral route, it calculates the influence of other users through a kind of hydromechanics algorithm-FluidRating. The final model we called ELMFluid which can express the flow of psychological energy through thermodynamics and hydromechanics. The experiments are performed using two real datasets in the end. The results show that the new model is superior to other algorithms.
\end{abstract}

\section{Introduction}

In modern age, more and more people have joined websites that provide product comparison information in order to obtain personalized recommendation services [1]. The most popular are based on trusted relationships, such as Epinions and Ciao [2]. There are two important functions of this type of service system. One is that the registered users can add other users into their trusted user list and the other is that all registered users can comment on a certain product and give a one- to five-star rating. These reviews and scores are provided for those who have not purchased as a judgment basis of their purchase.

Predecessors have already done a lot of work on recommendation systems based on trusted relationships. Two of the most classic models TidalTrust and MoleTrust are proposed by Golbeck and Massa, respectively [3, 4]. However, these models are still looking for general rules and do not go into the users' cognitive process. What is the real driving force of human behavior? Sigmund Freud thought it was "sex" and he began to introduce the model of the energy of physics into psychology [5]. Carl Gustav Jung developed this theory and used the concept of mental energy directly. Mental energy can do psychological work and produce people's perceptions, cognitions, emotions, thoughts, personality, and so on. In the field of market and psychology, many researchers have done some work in recent years, including finding the relationship between the user perception and content, usability and aesthetics of the online website [6,7], social selection mechanisms [8], the consumer mindset metrics [9], and so on. What we need to do is apply the existing psychological conclusions.

So, in order to explore user behavior from a deeper level, we generalize a classic market model-Elaboration Likelihood Model (ELM) [10, 11]. The model divides the user's mental state into central route and peripheral route. That is, whenever a user generates an attitude to a product, this user is affected by two aspects at the same time. On the one hand, it is the rational thinking about the value of goods. This part is the central route. The factors that work on the other hand include emotional factors, the expression of information, and so on. This part is the peripheral route. The first step of the model is to distinguish the user's motivation and ability to analyze and comprehend information. When the user has a high degree of processing and digesting the known information, the user's rational thinking in the central route will play a leading role. Otherwise, the peripheral route plays a leading role. For 
example, if a product is a celebrity endorsement which the user likes, the reason why the user likes the product is the emotional factors so the peripheral route is more effective. If a user is a digital product master, his own subjective opinions are dominant because he is well aware of the configuration information. This time the central route is more effective. On this basis, we express these two routes in the form of fluid mechanics from the idea of FluidRating $[12,13]$. Finally, the model was proposed, which we called ELMFluid.

Our new method makes the following contributions:

(1) The new model describes the user's internal psychological state and behavioral dynamics. This new model uses fluid mechanics and thermodynamics to express the transmission of mental energy.

(2) In the new model, we use the number of review items which indicates how deeply the user relies on the website and has processed the known information.

(3) ELMFluid is evaluated by using real datasets of Epinions.com and Ciao.com. The results show that the method is very efficient and effective.

The remaining part of the article is organized as follows:

The second part of the article investigates the relevant work. The solution section describes the problem and explains and extends the classic model. Then, a hypothesis about the new model is made and the performance of the algorithm is analyzed. The experimental part includes a wide range of experiments driven by real datasets to verify the hypothesis. In the final section, the article is summarized and future work is given.

\section{Related Work}

In this section, we introduce the previous work on the classic consumer research models, trust models and give the differences between the previous work and our model.

2.1. Consumer Research Model. The classic consumer research models are mostly based on psychology and sociology. Kahle et al. proposed a conceptual model of regional consumption differences, known as the TOFA (Traditionalism, Optimism, Fashion and Financing, Advance) model. The model uses the two indices $S$ (Style) and $R$ (Risk) to divide the consumers into four types and identify the changes in the basic style and the form of regional consumption [14]. Arnold Mitchell designed Values and Lifestyle Systems (VALS system). VALS combines Maslow's hierarchy of needs with the internal drivers and external drivers which are proposed by the American sociologist David Riesman. Nine lifestyle groups are identified, which have now been upgraded to VALS2 and divided into eight markets [15]. Based on value theory, Sheth, Newman, and Gross propose that products provide customers with five values. These are functions, emotions, conditions, society, and cognition. In different consumption context, consumers are affected differently by different values [16]. Most of the methods are based on statistics to classify the customers directly. The model used in this article is the Elaboration
Likelihood Model (ELM) by the psychologists Petty and Cacioppo [10, 11].

2.2. Trust Model. At present, most of the recommendation systems are based on the relationship graph. The most important step is to calculate the relationship value or trust transfer rules [17, 18]. Among the trust models, two wellknown models are TidalTrust based on breadth-first search proposed by Folbeck and MoleTrust proposed by Massa. TidalTrust is a method based on the shortest path, with high scalability and low complexity [3]. MoleTrust calculates the spread range of trust relationships by figuring out the central node [4]. Personalized PageRank calculates the trust value of the whole network node via Markov chain [19, 20]. Ziegler and Lausen used the local group trust propagation mechanism of the activation diffusion mechanism in psychology to calculate the trust value [21]. Kuter et al. predict the probability of trust among Bayesian network nodes by using probabilistic sampling techniques [22]. Liu et al. divide trusted users into different fields to improve recommendation accuracy [23]. Asghari and Azadi proposed an inverted ant colony optimization algorithm to find the reliable propagation path [24]. Deng used deep learning with the community effect for recommendations [25]. Ghavipour and Meybodi proposed DLATrust which is a learning automaton for discovering reliable paths [26]. However, most models do not take time evolution into account. FluidRating contacts the temperature and the user's rating by the dynamics theory [13]. The model we proposed considers the user's internal deeper and inner psychodynamics. In order to explore the user's behavioral motivation, the model considers the user's own inherent attitudes and personality (central route) and the part affected by other users (peripheral route). The new model also conforms to the general principle of transitivity.

2.3. Personality. The psychologists believe that personality is the driving force of behavior [27]. Personality is defined as a series of habitual behaviors evolved from biological and environmental factors, cognitive and emotional patterns. It is the stable difference between different people $[28,29]$. It also can have an impact on online behavior [30]. Through the study of personality traits, the online behavior of users can be well analyzed. For example, Feng uses personality traits to predict customer satisfaction and loyalty to the service companies through online replies [31]. Caci et al. analyze the use of Facebook by 600 users through personality traits [32]. Cojocaru et al. analyze social influence based on personality traits [33]. Hamburger believes that based on the Big Five personality, men with extraversion personalities prefer to use the leisure and entertainment services on the internet. Women with extraversion personality are more reluctant to use social networking services [34]. Most of the judgments on the user's personality are based on the text information posted by the user on the internet. When the website has only scores and no comments, it is very difficult to identify the user's personality, so at this point, our method only considers the user's rating habits about the product. 


\section{Preliminary}

3.1. FluidRating. The idea of FluidRating is an important part of our model $[12,13]$. For a certain product, FluidRating divides the users into two categories. One is the user who has already given an opinion on the product (defined as raters, denoted as $R$ ) and the other is the user who has not given an opinion (nonraters, defined as $N$ ). First, FluidRating builds the relationship net shown in Figure 1. It can be seen that raters $a_{1}$ and $a_{2}$ give 3 and 5 stars for the product. Raters $a_{3}, a_{4}$, and $a_{5}$ are nonraters. The trust relationships are represented by the arrows. User $a_{3}$ trusts user $a_{1}$, so user $a_{1}$ points to user $a_{3}$. A system is built based on a well-constructed net. Then, users are mapped into different containers. The trust relationship is mapped to a one-way channel. The temperature of the liquid in the container represents the user's opinion.

Finally, the liquid in containers $a_{1}$ and $a_{2}$ will flow to $a_{3}$, $a_{4}$, and $a_{5}$, and the temperature of the fused liquid from different-source containers represents the predicted rating.

3.2. ELM. The basic principle in ELM is shown in Figure 2. The proposed method simulates the user's thinking process and expresses that different persuasion routes play different roles. One is the central route and the other is the peripheral route. When users process information deeply, the proportion of the central route is more effective. On the other hand, when users think about the known information very lightly, the peripheral route is more effective [16]. Because each user's dependence on the website is different, this model provides great help for us to explore the psychological processes of different users.

Our new method configures two routes by using the product ratings and trust relationships on the website rather than the traditional cumbersome psychological measures methods in the form of questionnaires.

\section{Solution}

4.1. Development of the Classical Model and Hypotheses. According to the classical theoretical frameworks, we propose an improved model. We fuse FluidRating and personality theory in ELM as shown in Figure 3. For a product, each user's attitude is divided into two parts. One part is the central route generated by the user after the consideration of the information. In this article, we use the personality theory to solve this part. The other part is the attitude generated by users who are influenced by the reviews of users they trust, calculated using FluidRating, which is the peripheral route. Then, we take the number of user reviews as the proportion of the two parts, which could denote the degree of dependence of the website. Under the circumstances without text information, we classify users into four types by the reviews. Different types of users are affected by two routes differently. Finally, the method proposes a representation of fluid mechanics and thermodynamics that facilitates the computation of the mix and interaction with mental energy.

The psychological state of user $x$ or the user's comprehensive mental state is described as follows:

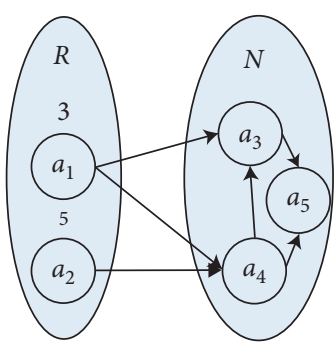

(a)

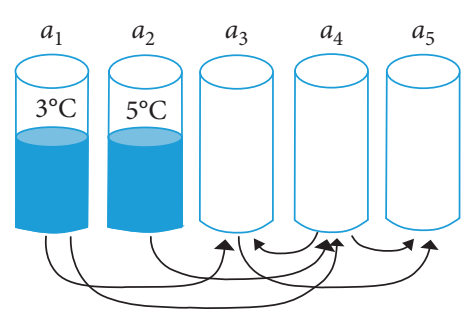

(b)
FIgURE 1: An illustration of a rating network and a FluidRating system: nodes represent users; numbers on nodes represent ratings given by users; directed edges represent the relations.

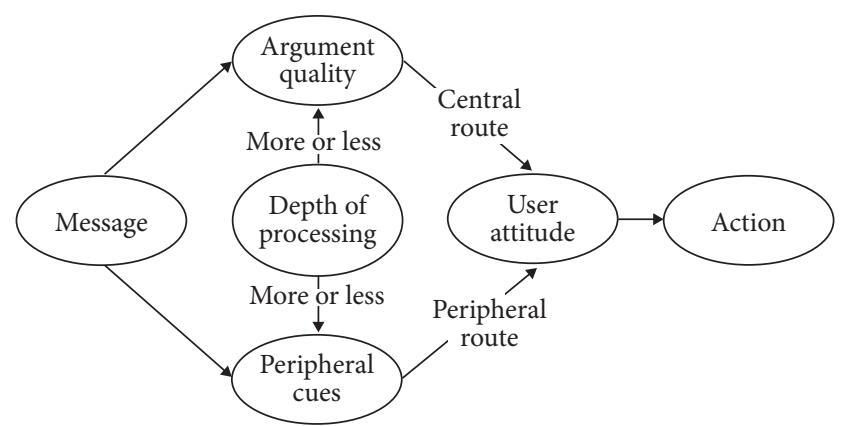

FIgURE 2: The Elaboration Likelihood Model.

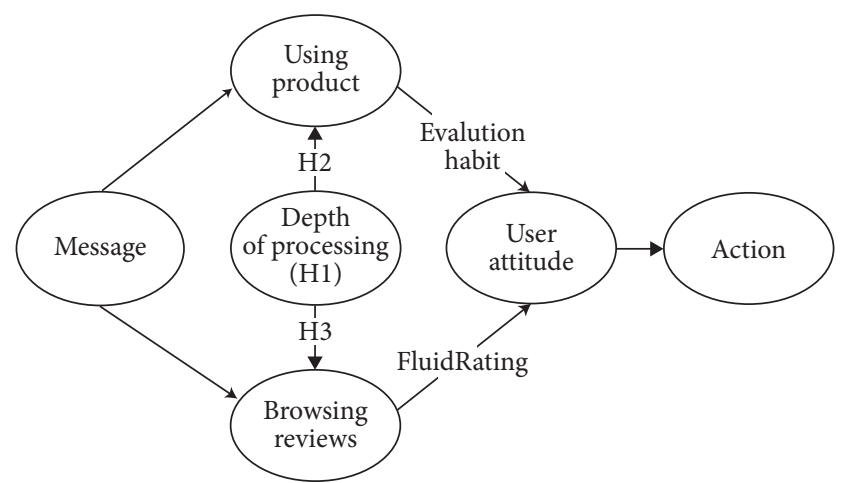

FIgURE 3: The ELMFluid model.

$$
\frac{\mathrm{d} x}{\mathrm{~d} t}=F(x)+\sum_{y=1}^{N} A_{x y} G(x, y),
$$

where $F(x)$ is the central route of user $x$ and $\sum_{y=1}^{N} A_{x y} G(x, y)$ is the peripheral route of user $x . G(x, y)$ is the effect of user $x$ receiving external factors $y$, like other users' ratings. $A_{x y}$ is the weight of the factor.

Through the above model, we make the following hypotheses:

H1: the more reviews the user has, the more the user can be affected by the peripheral route.

When a user evaluates more products, it proves that the user is also more likely to rely on the evaluation system and consider others' reviews. On the contrary, if a user 
has fewer evaluations, the user has deeper processing and his judgment is not easily affected.

$\mathrm{H} 2$ : evaluation habit is stable.

If a user's personality trait is that he often gives a rating higher or lower than the average rating, he will maintain this characteristic for a certain period of time. The formal representation is shown as follows:

$$
\begin{aligned}
F(x) & \approx \beta_{x} \cdot \overline{\text { rating }}, \\
\beta_{x} & =\frac{\sum_{n=1}^{N}\left(\text { rating }_{n(x)}-\overline{\text { rating }_{n}}\right) / \text { rating }_{n}}{N},
\end{aligned}
$$

where $\overline{\text { rating }}$ is the average rating of the $n$th product, rating $_{n(x)}$ is the rating of user $x$ of the $n$th product, and user $x$ rates $N$ products in all. $\beta_{x}$ represents the deviation degree between the evaluation of user $x$ and the average opinion. We believe that the bias $\beta_{x}$ of each user in the evaluation is stable.

H3: a user can be impacted differently by the users with different influence.

$$
\sum_{y=1}^{N} A_{x y} G(x, y)=\gamma_{x y} \text { FluidRating }(x, y),
$$

where FluidRating $(x, y)$ is the peripheral route based on FluidRating. $\gamma_{x y}$ is the parameter about the influence of different users.

Finally, based on the above hypotheses, we construct the ELMFluid, as shown in Figure 3.

4.2. Problem Formulation and Overview of the Model. Because the system is an idealized model, we make the following provisions:

Assumption 1. The system is a closed system; the entropy of the system is constant.

Assumption 2. The temperature in the container will not be changed until there is liquid exchanging in the container.

As in FluidRating, the new model is divided into 3 parts: one-way connection pipes, fluid, and containers. In our model, the container is used to represent each node in the relation graph and assume that the container is large enough. The bottom of the containers is at the same level so that liquid in the container has the same potential energy. The temperature of the fluid indicates users' attitudes. There is no liquid in containers of nonraters before the system initializes because the nonraters have not given the rating yet. The one-way pipe represents the trust relationship and this part is the peripheral route.

We added one extra pipe to each container. The temperature of this liquid represents the self-generated internal attitude of the user, and that is the central route.

\subsection{Algorithm Details}

4.3.1. Parameter Initializing. When the model starts, the fluid height in the container $h$ and the cross-sectional area $b$ will be given. Until the empty container is filled with liquid, we divided the process of liquid exchange into $k$ time slots from the start.

4.3.2. Fluid Updating Execution. The updating process is shown in Figure 4 and the update of the liquid volume between the adjacent containers is computed by the Bernoulli formula in physics. The updated volume $S_{a a^{\prime}}(i)$ from container $a$ to container $a^{\prime}$ at ith time slot is shown in (5). This part can also be understood as the volume in the pipe from container $a$ to container $a^{\prime}$.

$$
S_{a a^{\prime}}(i)=2 g \sqrt{h_{a}(i)-h_{a^{\prime}}(i)} \times \Delta t .
$$

Here, $g$ is the constant gravitational acceleration. $\Delta t$ is the time gap and $h_{a}(i)$ and $h_{a^{\prime}}(i)$ are the fluid height in containers $a$ and $a^{\prime}$.

The volume of the fluid in a certain container is the remaining $S_{a}(i)$ plus $\sum_{a^{\prime \prime} \in N_{a}^{\text {in }}} S_{a^{\prime \prime} a}(i)$ which is the fluid flowing in from each container which has the height difference and minus $\sum_{a^{\prime} \in N_{a}^{\text {out }}} S_{a a^{\prime}}(i)$ which is the fluid flowing out at the next time slot. So, the volume $S_{a}(i+1)$ in container $a$ at the $i+1$ th time slot could be figured out by

$$
S_{a}(i+1)=S_{a}(i)-\sum_{a^{\prime} \in N_{a}^{\text {out }}} S_{a a^{\prime}}(i)+\sum_{a^{\prime \prime} \in N_{a}^{\text {in }}} S_{a^{\prime \prime} a}(i) \text {. }
$$

The temperature of the liquid in the container is calculated by the formula which figures out the temperature of the mixed liquid. $t_{a}(i+1)$ is the temperature of the liquid in container $a$ at the $i+1$ th time slot. $t_{a^{\prime \prime} a}$ is the temperature of the liquid flowing from container $a^{\prime \prime}$ to $a$.

$$
t_{a}(i+1)=\frac{t_{a}(i) \cdot\left[S_{a}(i)-\sum_{a^{\prime} \in N_{a}^{\text {out }}} S_{a a^{\prime}}(i)\right]+\sum_{a^{\prime \prime} \in N_{a}^{\text {in }}}\left[t_{a^{\prime \prime} a}(i) \cdot S_{a^{\prime \prime} a}(i)\right]}{S_{a}(i+1)} .
$$

4.3.3. Time Complexity Analysis. The algorithm is shown in Algorithm 1. Lines from 1 to 10 show the discrete process to figure out the liquid flowing in with $k$ time slots. From line 2 to 6 , we figure out the updated fluid which should flow to each nonrater's container in each time slot. From line 7 to 9 , we maintain the fluid height and temperature of the raters' containers. Each round of fluid updating from line 1 to 10 takes a time complexity of $O(V+E)$. So, the total time complexity of the algorithm in $k$ time slots is $O(k *(V+E))$. Finally, in line 11 , we get the predicted rating represented by the temperature. 


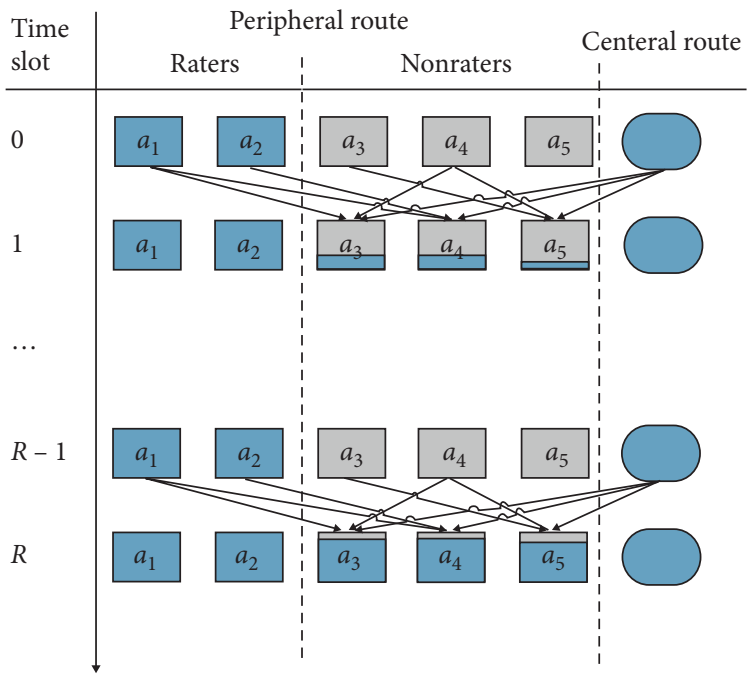

FIGURE 4: The iteration of the model.

Input: The rating network $G$; The parameters for the initialization $p$; $K$ time slots Output:The predicted ratings of the target non-raters; Initialize:

(1) Associate each node in $G$ with a container and each edge with a directed pipe;

(2) Add an extra pipe that can inject fluid in all containers;

(3) Initialize the fluid height and temperature in each container;

(1) for $i=0$ to $K$ do

(2) for each non-rater's container do

(3) if $h_{a}(i)>h_{a}(i)$ then

(4) Update the volume and temperature according to equations (6) and (7);

(5) end if

(6) end for

(7) for each rater's container do

(8) Inject additional fluid to maintain the fluid height and temperature;

(9) end for

(10) end for

(11) return the fluid temperature in the target container.

Algorithm 1: ELMFluid ( $G, p, K)$.

Above all, ELMFluid could be very efficient for realworld rating predictions involving millions of users because of the sparse network.

\section{Evaluation}

\subsection{Experimental Design}

5.1.1. Datasets. The datasets we used are from Epinions.com and Ciao.com. These two websites are very popular about the consumer reviewing [35]. Visitors can browse a variety of comments to help them make the right choice for shopping based on trust relationship. The Epinions dataset is a classical set, which is published by Massa and Avesani [4]. There is a total of 49,290 users with 487,181 trust relationships and a total of 139,738 different products with 664,824 reviews. Another dataset is crawled from the Ciao.com by Tang Jiliang. There are more than 57544 relationships on 2378 users and nearly 284086 ratings of 16,861 products. The basic information of two datasets is shown in Figures 5-7. The Epinions and Ciao data used to support the findings of this study are included within the Supplementary Material. Both datasets can also be downloaded form http://www.cse.msu. edu/tangjili/ [36] and for the reason of privacy protection, the original user ID has been processed and hashed.

The rating distribution of two datasets is shown in Figure 5.

It can be seen that most of them are concentrated on four stars and five stars. Users prefer to give praise. This appeals to common sense.

The distribution of the reviews is shown in Figure 6. The number of users with most reviews is lower than any other user group.

The distribution of the followers of the user is shown in Figure 7. Half of the users on Epinions have more than 15 followers and half of the users on Ciao have more than 30 followers. 


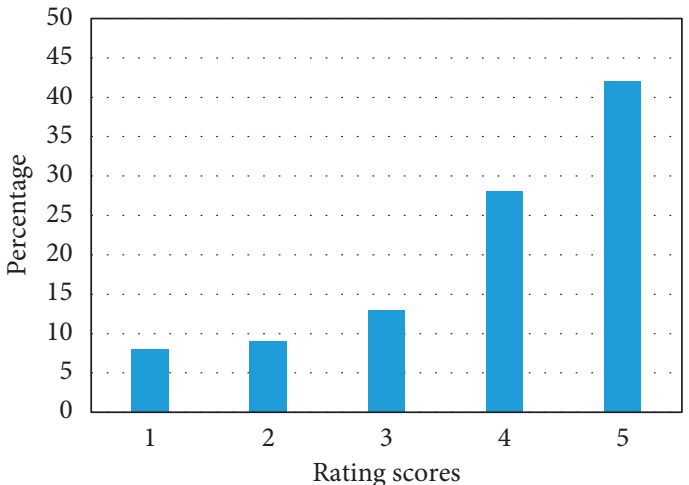

Rating distribution

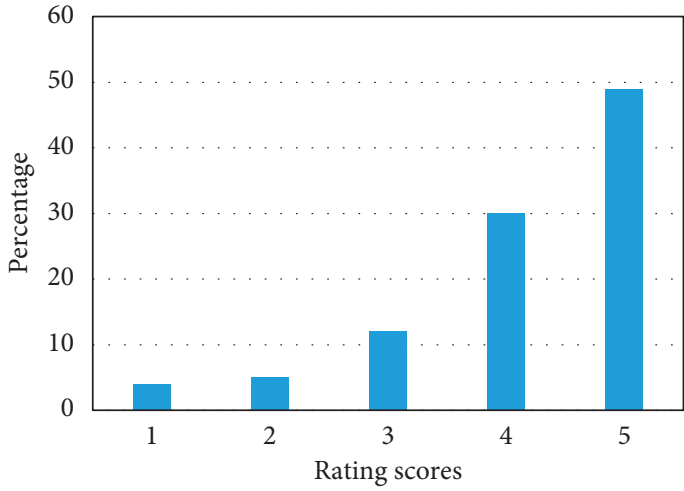

Rating distribution

Figure 5: The distribution of the rating scores. (a) Epinions dataset. (b) Ciao dataset.

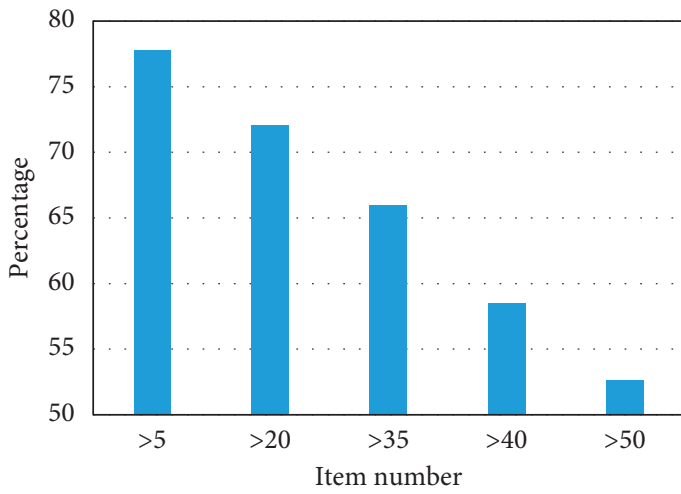

Item distribution

(a)

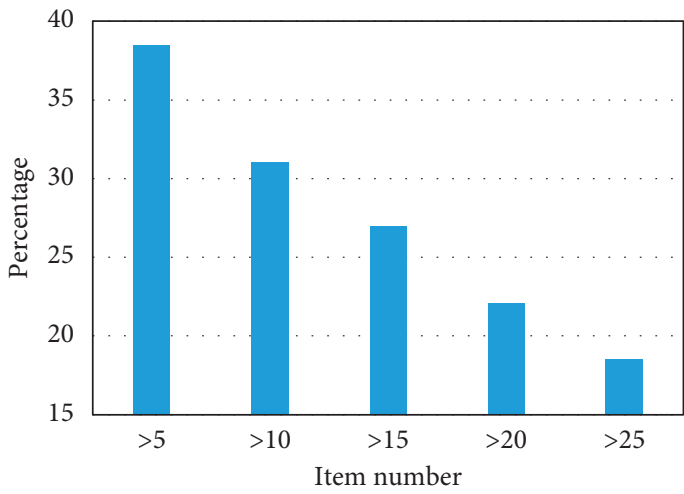

Item distribution

(b)

Figure 6: The distribution of the reviews. (a) Epinions dataset. (b) Ciao dataset.

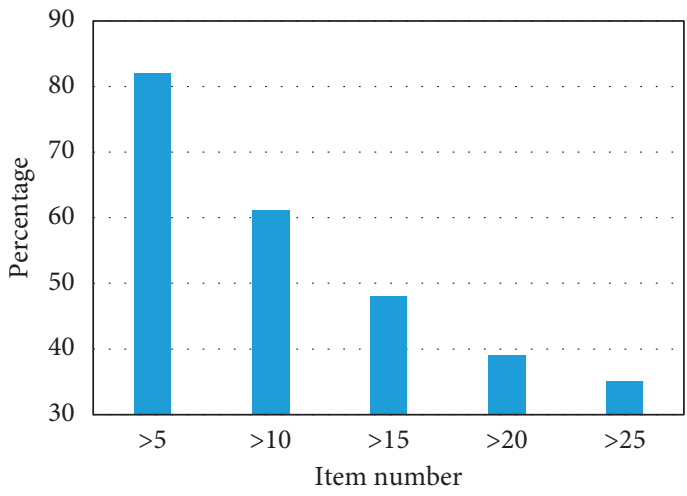

Follower distribution

(a)

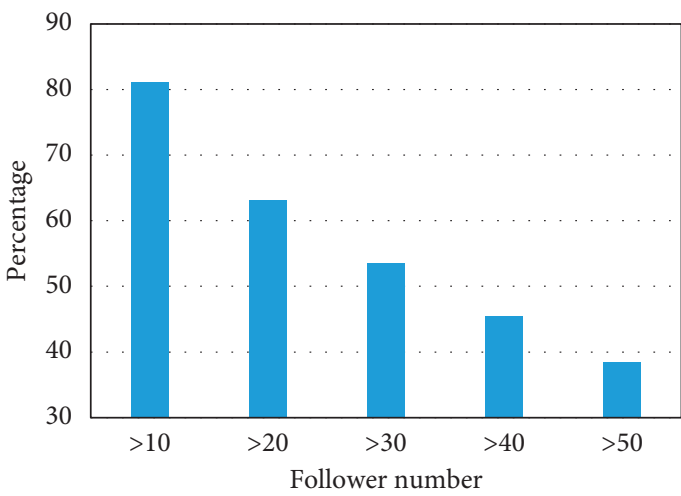

Follower distribution

(b)

Figure 7: The distribution of the followers. (a) Epinions dataset. (b) Ciao dataset. 
It can be seen that the users of Ciao pay more attention to the trust relationship and users of Epinions pay more attention to the reviews. The users in Epinions usually give more reviews than Ciao and the users in Ciao follow more other users than Epinions.

5.1.2. Evaluation Method. To measure the prediction performance of the ELMFluid, we use the leave-one-out method $[4,37]$. For the subgraph of a user, the true rating of that user is masked and then it will be predicted based on the subgraph. Then, the prediction error is the difference between the predicted rating and the masked rating.

In order to verify the propagation ability of the algorithm, for the target user, we tested three different calculation scopes of a target user. As is shown in Figure 8, the 1hop subgraph only considers the target user and his followees with ratings. Then, we add all the followees of the target user into the 2-hop subgraph and if a followee of these has not given a rating, we also add his followees. The 3-hop subgraph is the next extension layer of the 2-hop subgraph.

The process of simulation is also very simple. Initialize the model based on the parameters in the table. The fluid height in the container is 10 and the cross-sectional area is 1 . Then update the exchanging liquid according to the algorithm. We divided the process of liquid exchanging into 20 time slots and the predicted values (temperature values) of each time slot were recorded. Finally, we get the attitude of the target user.

Table 1 shows the parameter settings.

5.1.3. Accuracy Metrics. The accuracy evaluation methods we used are RMSE and F1-score [38].

Root Mean Square Error (RMSE) is a commonly used measure of the difference between the samples and total values.

$$
\mathrm{RMSE}=\sqrt{\frac{\sum_{i=1}^{N}\left(X_{\mathrm{obs}, i}-X_{\text {model }, i}\right)^{2}}{N}},
$$

where $N$ is the total number of user/item pairs which need to be predicted and $X_{\mathrm{obs}, i}$ and $X_{\text {model, } i}$ denote the real ratings and predicted ratings.

The second performance metric is the classic F-score, which is the harmonic mean of precision and recall:

$$
F-\text { score }=\frac{2 \mathrm{TP}}{2 \mathrm{TP}+\mathrm{FP}+\mathrm{FN}} \text {. }
$$

FN (the false-negative case) is the number of incorrect predictions when the ground truth is that the corresponding user has a rating score of lower than three (called negative rating). TP and FP (the true and the false-positive cases) are the numbers of correct and incorrect predictions, respectively, when the ground truth is that the corresponding user has a rating of no less than three (called positive rating).

A smaller RMSE indicates a better prediction. A larger $F$ score indicates a better prediction. So, when we get the prediction evaluation with a smaller RMSE value and a larger $\mathrm{F}$-score at the same time, this shows that they are consistent, and the method is effective.

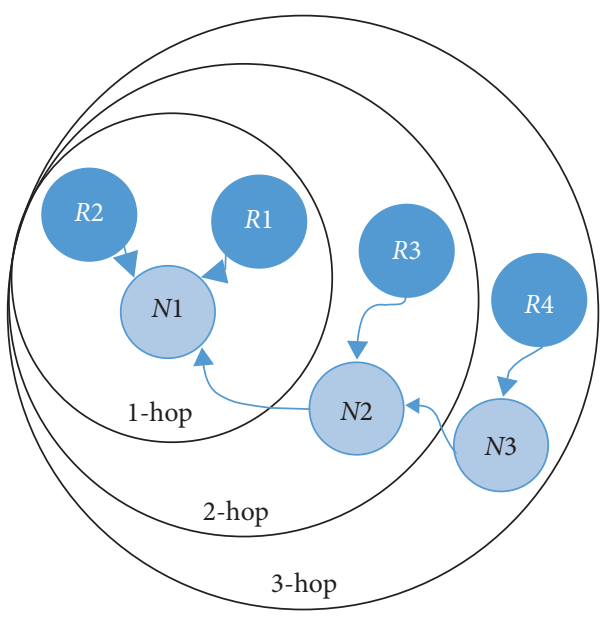

FIgURE 8: The scope of different hops.

TABle 1: Parameter settings.

\begin{tabular}{lcc}
\hline Parameter & Description & Default value \\
\hline$h$ & Fluid height & 10 \\
$b$ & Cross-sectional area of containers & 1 \\
$k$ & Round number & {$[1,20]$} \\
$\Delta$ & Time gap, length of one time slot & 0.05 \\
& Default central route & $50 \%$ \\
& Default peripheral route & $50 \%$ \\
\hline
\end{tabular}

\subsection{Experimental Results and Analysis}

5.2.1. Impact of the Process Depth. In this part of the experiment, we compare the results of four runnings of our algorithm with different values of the central and peripheral routes. First, the central route and the peripheral route are the same. Second, the percent of the central route is larger than the peripheral route. The percentage of central route is 60 and the percentage of the peripheral route is 40 . Third, the percentage of central route is 40 and the percentage of the peripheral route is 60 . Last, we divide users into four types by the number of reviews and we set different parameters to different users as in Table 2 to show the personal processing depth.

The comparison of the results in Figures 9 and 10 shows that the personal processing depth is the most accurate for the highest $F$-score and smallest RMSE. These results meet hypothesis $\mathrm{H} 1$. The accuracy is $5 \%$ higher than the default model in each subgraph from RMSE, followed by the large proportion of the central route and then the small proportion and we can draw the same result from F-score. Under the similar recommended website based on trust relationship, the user's central route is more important than the peripheral route because the prediction of a larger central route is more accurate than others except for the personalized process.

From the subgraph of different hops to analysis of the results, the 1-hop works best on the Epinions dataset, and the third hop results from the Ciao dataset are the best. The results on both datasets are almost identical and the nuances can be due to user's different concerns because the results of three subgraphs are very close. 
TABLE 2: Personal parameter settings dataset.

\begin{tabular}{lcc}
\hline Number of reviews & Central route (\%) & Peripheral route (\%) \\
\hline$x<10$ & 20 & 80 \\
$10 \leq x<20$ & 40 & 60 \\
$20 \leq x<30$ & 60 & 40 \\
$30 \leq x<40$ & 80 & 20 \\
\hline
\end{tabular}

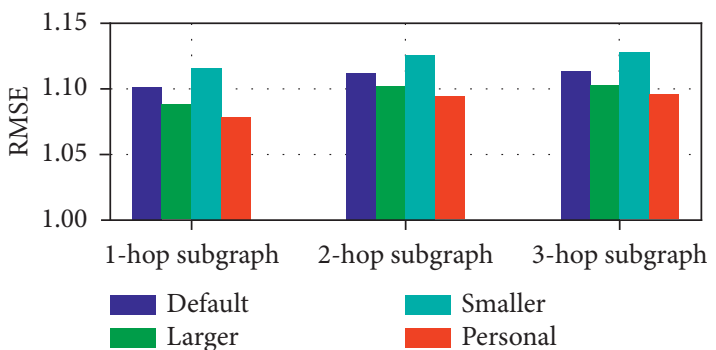

(a)

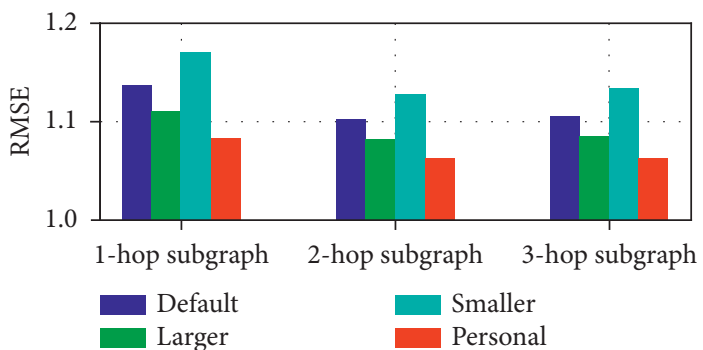

(b)

Figure 9: Comparison of different depth of processing, in terms of the RMSE metric. (a) The RMSE in the Epinions dataset. (b) The RMSE in the Ciao dataset.

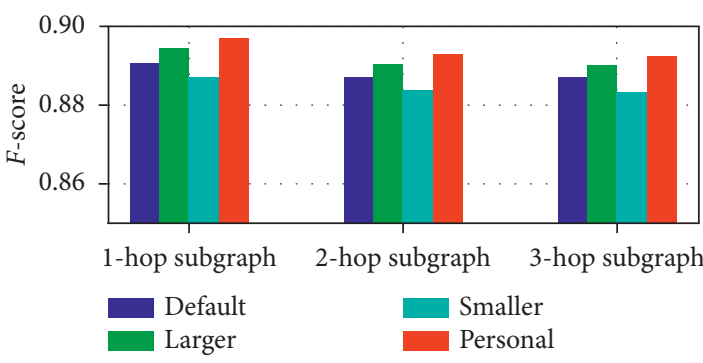

(a)

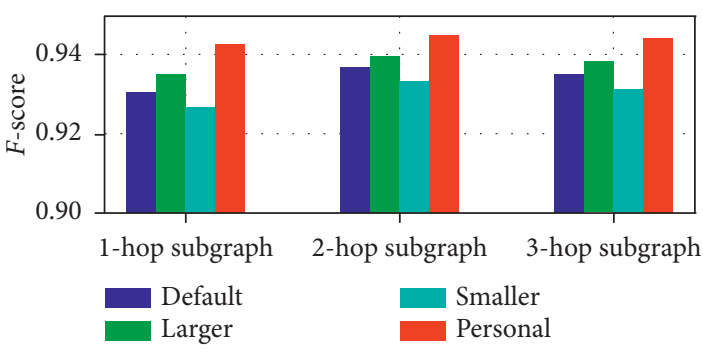

(b)

Figure 10: Comparison of different depth of processing, in terms of the F-score metric. (a) The F-score in the Epinions dataset. (b) The Fscore in the Ciao dataset.

5.2.2. Impact of the Personality. The second experiment compares the default central route and the personalized central route which considers the personality. The first result is based on the default model and the second is based on the personalized rating preference which improves the central route of the model.

The results in Figures 11 and 12 show that the model with user's personalized scoring habits is more effective and the two datasets produced similar results. The RMSE with personal improvement in both datasets is smaller than the default and the F-score is higher. In the Epinions dataset, three conditions with different hops are similar. The 2-hop and 3-hop on the Ciao dataset are better than the first. The accuracy of the personalized central route on both datasets is about $5 \%$ higher than the default, so we conclude that considering the preference of the user's scoring, the predicted result is more accurate. These meet hypothesis $\mathrm{H} 2$.

5.2.3. Impact of the Trust Degree. In this part of the experiment, we use the number of users' followers as personalized parameters. If the number of the followers of a user is greater than a common user, the temperature of the liquid flowing from the container represented by this user should be increased. When the user has fewer followers, the temperature should be decreased. In the process of parameter adjustment, if the coefficient of change is too small, the effect is not obvious. If the coefficient of change is large, the predictions are not good. So, we update the temperature by 10 , where $n$ is the number of followers. When the number of followers is higher, the number of this type of user is more sparse, so the logarithmic operation is added. The number of followers is less than 100 , so the final range of the coefficients is $[-0.1,0.1]$.

$$
t=t+t \cdot\left(\frac{\log (n)}{10-0.1}\right)
$$

The results are shown in Figures 13 and 14.

From the results in Figures 13 and 14, by using the current parameters, we can get a slight improvement. In each subgraph of the two datasets, we get a slightly smaller or equal RMSE compared with the default condition. The $F$ score value is similar with the RMSE value.

It is worth noting that when we get equal RMSEs, this prediction is also meaningful if the F-score value is larger. Or 


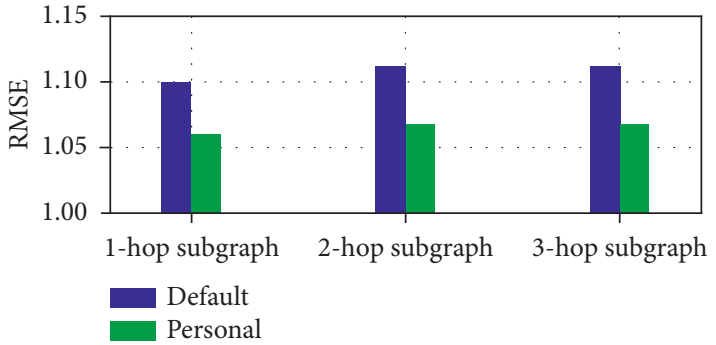

(a)

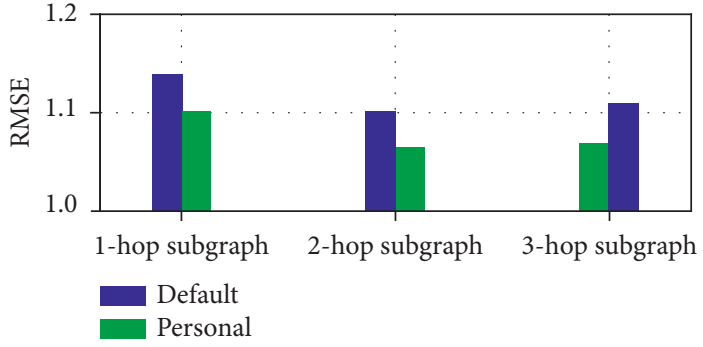

(b)

FIGURE 11: Comparison of default model and model with personality of scoring habits, in terms of the RMSE metric. (a) The RMSE in the Epinions dataset. (b) The RMSE in the Ciao dataset.

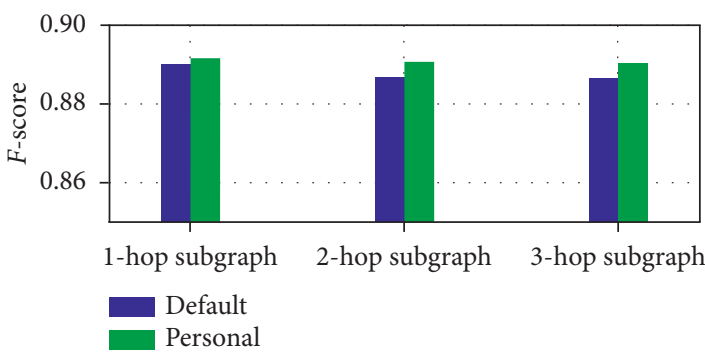

(a)

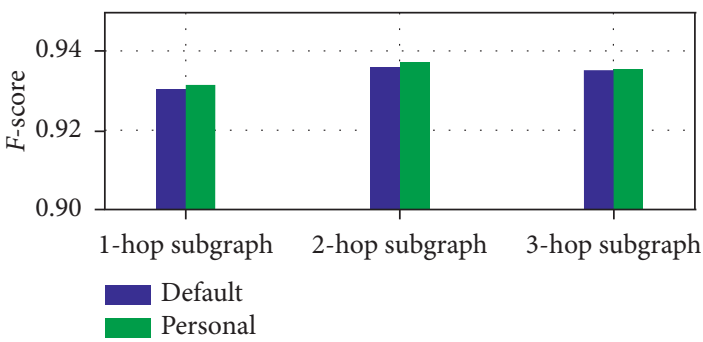

(b)

Figure 12: Comparison of default model and model with the personality of scoring habits, in terms of the F-score metric. (a) The F-score in the Epinions dataset. (b) The F-score in the Ciao dataset.

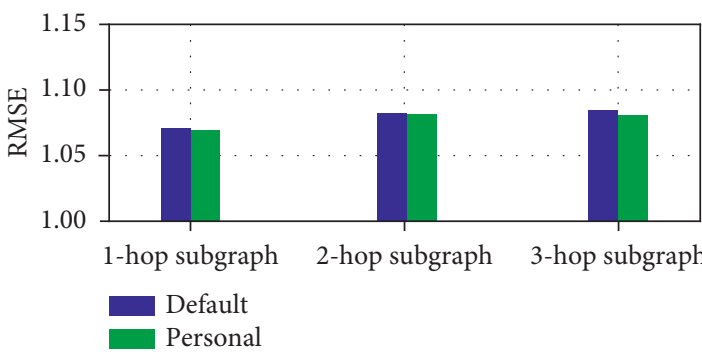

(a)

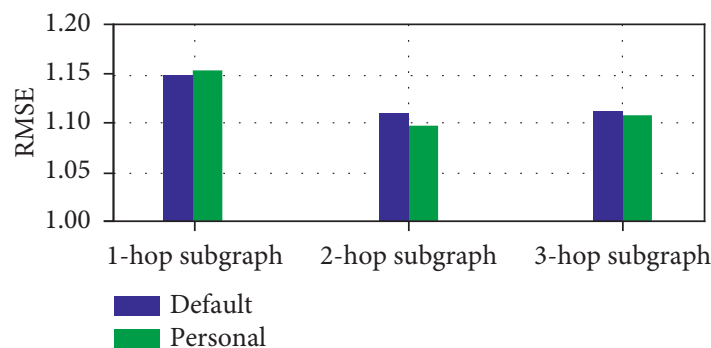

(b)

FIGURE 13: Comparison of the default model and model with trust degree, in terms of the RMSE metric. (a) The RMSE in the Epinions dataset. (b) The RMSE in the Ciao dataset.

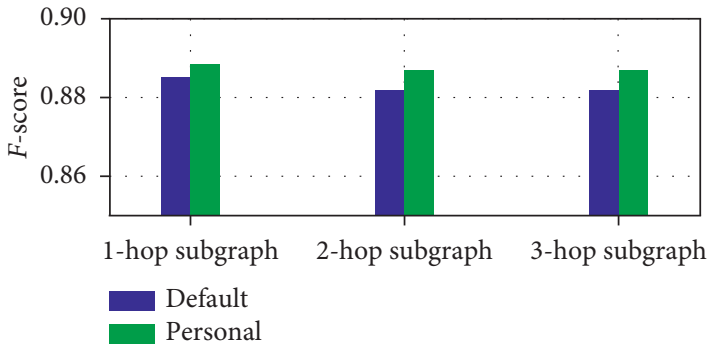

(a)

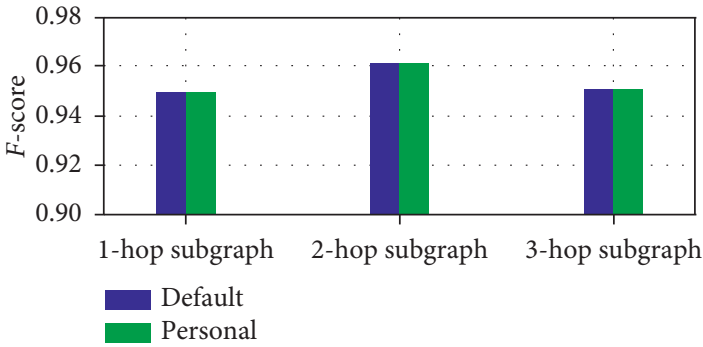

(b)

Figure 14: Comparison of the default model and model with trust degree, in terms of the F-score metric. (a) The F-score in the Epinions dataset. (b) The F-score in the Ciao dataset. 
if $F$-scores are equal, RMSE is smaller. The first case shows that although the deviation from our improvement is equal to the default method, we get truer positive cases, just like our results from the Epinions. The second case shows that when we get the same number of true positive cases, our prediction deviation is less from the ground truth, just like our results from the Ciao.

5.2.4. Multiple Algorithm Comparison. In this section, we tested the comparison among the ELMFluid model constructed by individual factors in the case of previously set parameters, every single factor, and other methods including the FluidRating model, the basic model of this article. Because a smaller RMSE means a better result, while a larger $F$ score means a better result, from the results in Figures 15 and 16 , the superiority of our algorithm has been clearly seen compared with the classical algorithms, including TidalTrust, MoleTrust, RandomWalk, PageRank, and FluidRating. On the three subgraphs with different hops, we reached a similar conclusion. From RMSE metrics, in the Epinions dataset, the accuracy of the user character role theory increased $10 \%$ than FluidRating and processing depth contribute 5\%. For the Ciao dataset, the accuracy improvements were $15 \%$ and $20 \%$, respectively. That means we have a smaller error. From the $F$-score metrics, we can come to the consistent conclusion with the RMSE metric that we have higher $F$-score values. That means we get truer positive cases.

5.2.5. A Deeper Discussion. In this part of the experiment, we tested the effect of time evolution from the 1st time slot to the 20th time slot on the model first. From the result in Figure 17, each iteration of the model has little impact on the model. The result has little change from the first time slot to the end.

Next, we conduct experiments on users with different attributes. In the Epinions dataset, we set up two subsets with the users whose followers are more than 10 and more than 20. In the Ciao dataset, two new datasets are made up of the users whose followers are more than 20 and 40, respectively.

Another experiment is also conducted under different subsets. In the Epinions dataset, users whose reviews are more than 20 and more than 40 will compose the two new datasets and the number of users' reviews of two new Ciao subsets is more than 10 and 20 .

The reason for dividing the new dataset in this way is that each subset accounts for $30 \%$ or $60 \%$ of the full dataset approximately, which is good for our comparison between the users with different attributes.From the first experiment, it can be seen in Figures 18 and 19 that in the Epinions dataset, the users with the number of followers greater than 20 are predicted the most accurately with the smallest RMSE and the highest $F$-score. Then, the users with the number of followers greater than 10 are the second and the complete dataset has the worst predicted results. We can draw a similar conclusion on the Ciao dataset and the little difference is that we get the lightly truer positive cases from the

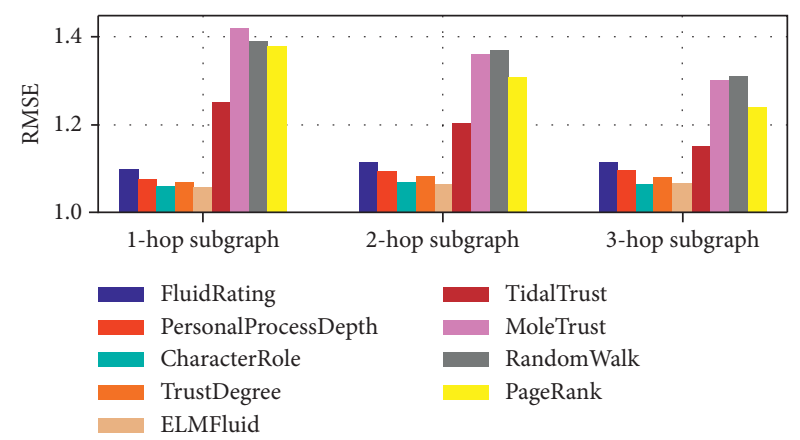

(a)

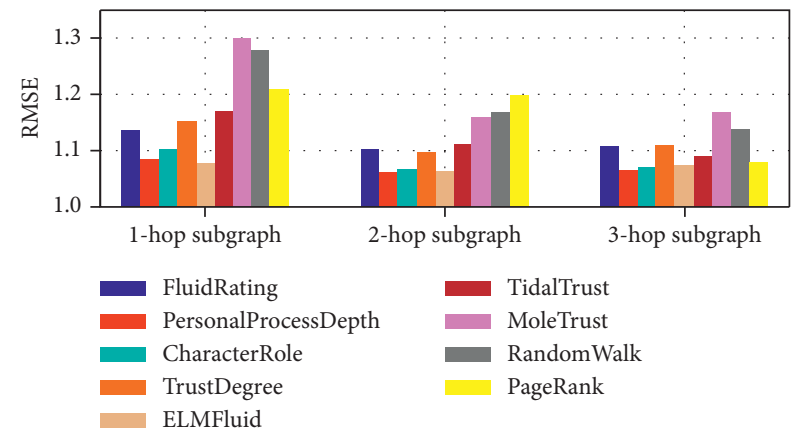

(b)

FIGURE 15: Comparison of different factors and classical methods, in terms of the RMSE metric. (a) The RMSE in the Epinions dataset. (b) The RMSE in the Ciao dataset.

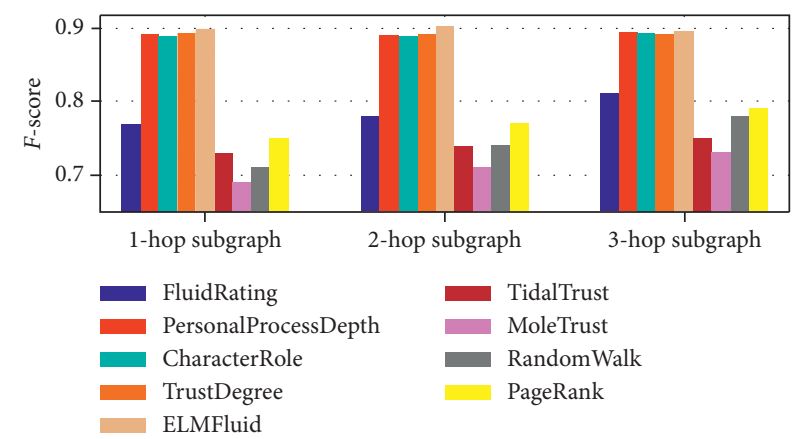

(a)

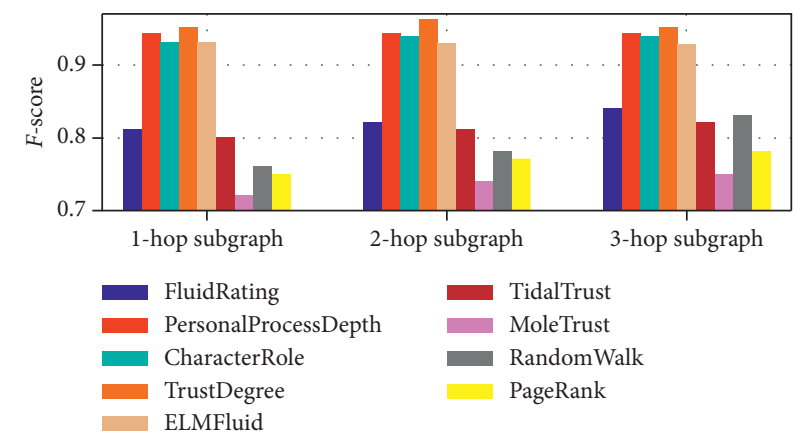

(b)

FIgURE 16: Comparison of different factors and classical methods, in terms of the F-score metric. (a) The F-score in the Epinions dataset. (b) The F-score in the Ciao dataset. 


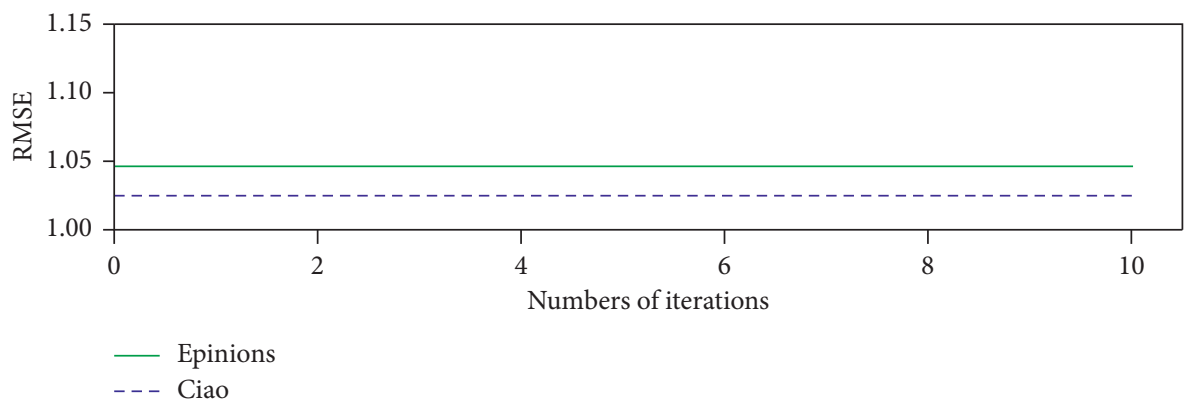

(a)

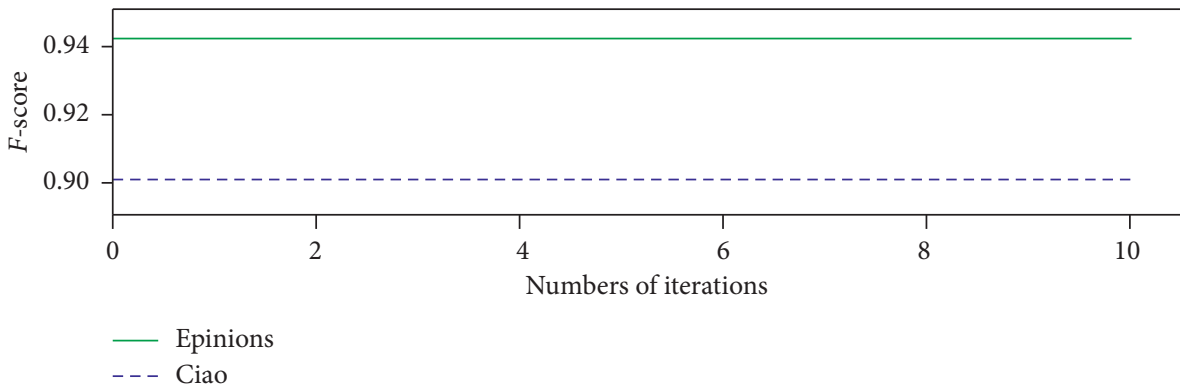

(b)

FIGURE 17: Impact of time evolution.

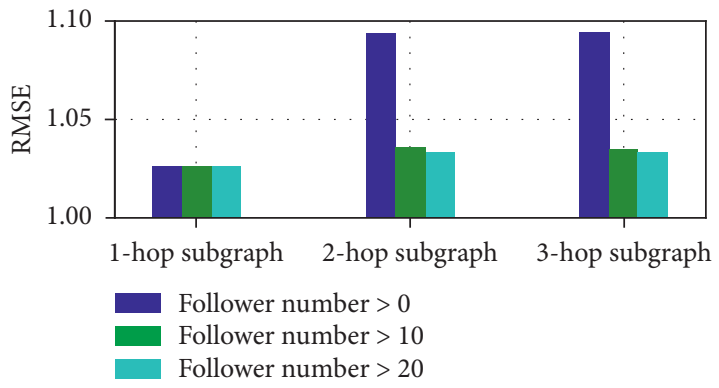

(a)

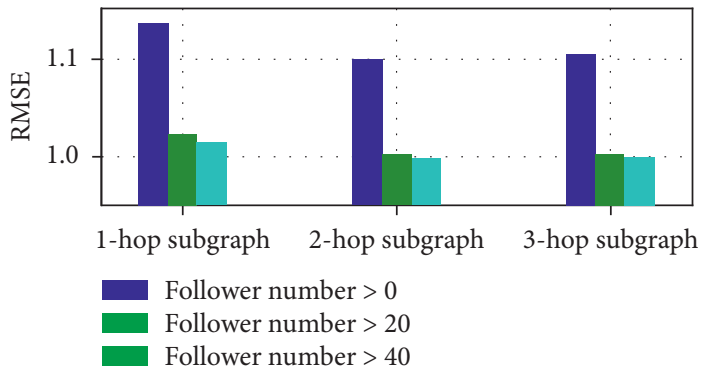

(b)

FIGURE 18: Impact of follower number, in terms of the RMSE metric. (a) The RMSE in the Epinions dataset. (b) The RMSE in the Ciao dataset.

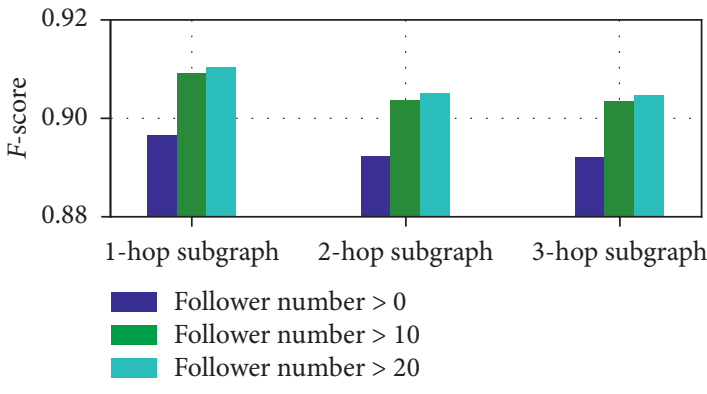

(a)

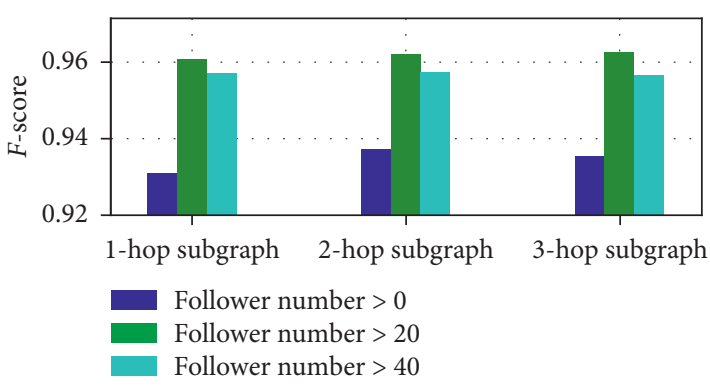

(b)

Figure 19: Impact of follower number, in terms of the F-score metric. (a) The F-score in the Epinions dataset. (b) The F-score in the Ciao dataset.

subdataset made up of the users with the number of followers greater than 20. So usually, the more followers the user has, the more accurate our model predicts.
The second experiment shown in Figures 20 and 21 studies the relationship between the model's accuracy and the number of user reviews. It can be seen in Epinions 


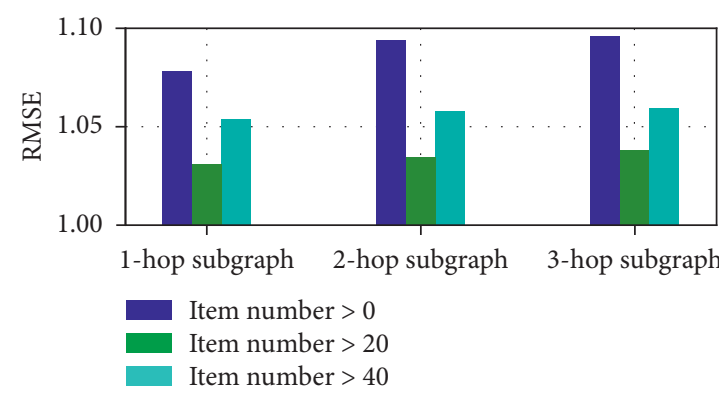

(a)

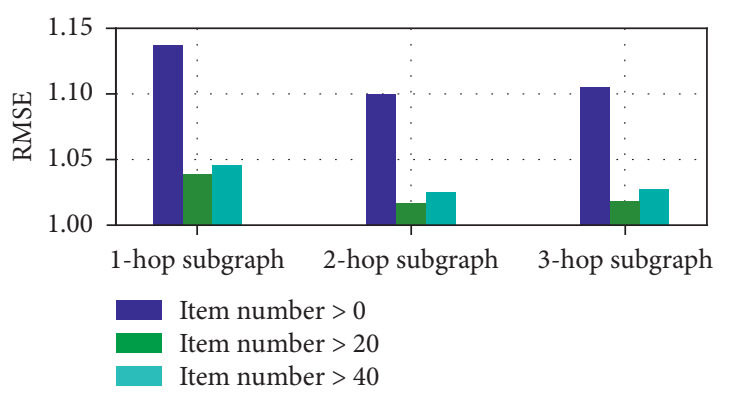

(b)

Figure 20: Impact of review number, in terms of the RMSE metric. (a) The RMSE in the Epinions dataset. (b) The RMSE in the Ciao dataset.

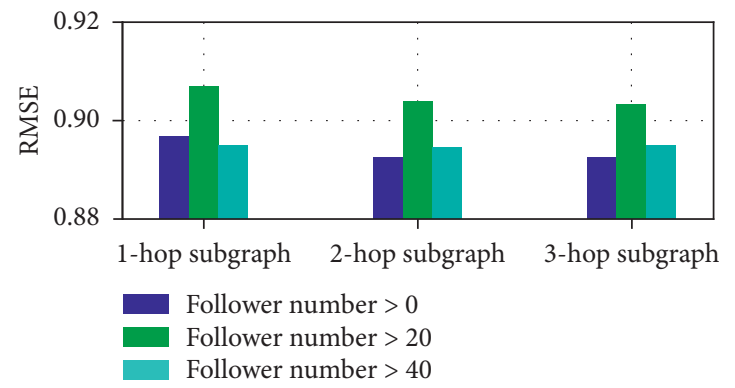

(a)

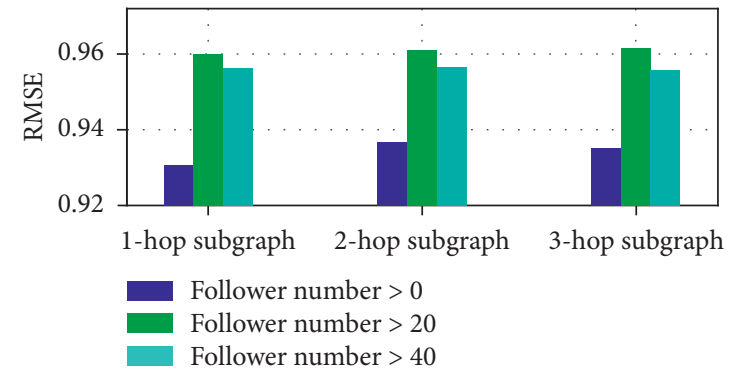

(b)

Figure 21: Impact of review number, in terms of the F-score metric. (a) The RMSE in the Epinions dataset. (b) The RMSE in the Ciao dataset.

dataset that, generally, users with more reviews will get smaller RMSE, higher F-score, so they can get more accurate prediction than users with fewer reviews, but when the number of reviews exceeds a certain range, the accuracy of the prediction result will be reduced. The results of the Ciao dataset also confirm this point.

\section{Conclusion and Future Work}

In this article, we combine the user personality theory and FluidRating as the central route and peripheral route of ELM. This method starts from the user's internal psychological structure so that it can better express the spread of mental energy through thermodynamics and fluid mechanics. The method is closer to the user's true psychological state, so it can predict more accurately and comprehensively. This paper verifies the rationality of the model and the validity of the hypothesis through experiments on two real datasets.

In future work, the model can be extended by the role of the user in the community, as well as the user's sense of efficacy in community, and the user's psychological process needs to be expressed with more accurate physical form. Other psychological concepts and analytical methods should also be introduced, such as the conformity biases and more detailed personality by the users' comments and the LIWC dictionary.

\section{Data Availability}

The data used to support the findings of this study are included within the article.

\section{Disclosure}

The funders had no role in study design, data collection and analysis, decision to publish, or preparation of the manuscript.

\section{Conflicts of Interest}

The authors declare that they have no conflicts of interest regarding the publication of this paper.

\section{Acknowledgments}

This paper was supported by the National Natural Science Foundation of China under Grants nos. 61672179, 61370083, and 61402126, the Research Fund for the Doctoral Program of Higher Education of China under Grant no. 20122304110012, the Youth Science Foundation of Heilongjiang Province of China under Grant no. QC2016083, Heilongjiang Postdoctoral Fund no. LBHZ14071, and the Fundamental Research Funds for the Central Universities no. HEUCF20181312.

\section{References}

[1] W. Li, Z. Ye, M. Xin, and Q. Jin, "Social recommendation based on trust and influence in sns environments," Multimedia Tools and Applications, vol. 76, no. 9, pp. 11585-11602, 2017.

[2] J. Tang, H. Gao, and H. Liu, "mtrust: discerning multi-faceted trust in a connected world," in Proceedings of the ACM 
International Conference on Web Search and Data Mining, vol. 93-102, Cambridge, UK, 2012.

[3] J. A. Golbeck, Computing and Applying Trust in Web-Based Social Networks, University of Maryland, College Park, MD, USA, 2005.

[4] P. Massa and P. Avesani, "Trust metrics on controversial users: balancing between tyranny of the majority and echo chambers," International Journal on Semantic Web and Information Systems, vol. 3, no. 1, pp. 39-64, 2007.

[5] S. Freud, Group Psychology and the Analysis of the Ego, Bantam Books, New York, NY, USA, 1965.

[6] T. T. Meinald, I. Blotenberg, and R. Jaron, "User evaluation of websites: from first impression to recommendation," Interacting with Computers, vol. 26, no. 1, pp. 89-102, 2018.

[7] S. Vilches-Montero, N. M. H. Nik Hashim, A. Pandit, and R. Bravo-Olavarria, "Using the senses to evaluate aesthetic products at the point of sale: the moderating role of consumers' goals," Journal of Retailing and Consumer Services, vol. 40, pp. 82-90, 2018.

[8] J. M. Davis and D. Agrawal, "Understanding the role of interpersonal identification in online review evaluation: an information processing perspective," International Journal of Information Management, vol. 38, no. 1, pp. 140-149, 2018.

[9] A. Colicev, A. Malshe, K. Pauwels, and P. O'Connor, "Improving consumer mindset metrics and shareholder value through social media: the different roles of owned and earned media," Journal of Marketing, vol. 82, no. 1, pp. 37-56, 2018.

[10] R. E. Petty and J. T. Cacioppo, The Elaboration Likelihood Model of Persuasion, Springer, Berlin, Germany, 1986.

[11] F. Marquart and B. Naderer, Communication and Persuasion: Central and Peripheral Routes to Attitude Change, Springer, Berlin, Germany, 2016.

[12] W. Jiang, J. Wu, G. Wang, and H. Zheng, "Fluidrating: a timeevolving rating scheme in trust-based recommendation systems using fluid dynamics," in Proceedings of the IEEE INFOCOM 2014-IEEE Conference on Computer Communications, pp. 1707-1715, Toronto, Canada, April 2014.

[13] W. Jiang, J. Wu, G. Wang, and H. Zheng, "Forming opinions via trusted friends: time-evolving rating prediction using fluid dynamics," IEEE Transactions on Computers, vol. 65 , no. 4 , pp. 1211-1224, 2016.

[14] L. R. Kahle, R. Liu, and H. Watkins, "Psychographic variation across the United States geographic regions," Advances in Consumer Research, vol. 19, no. 1, pp. 346-352, 1992.

[15] L. R. Kahle, S. E. Beatty, and P. Homer, "Alternative measurement approaches to consumer values: the list of values (lov) and values and life style (vals)," Journal of Consumer Research, vol. 13, no. 3, pp. 405-409, 1986.

[16] M. C. Gilly, J. N. Sheth, B. I. Newman, and B. L. Gross, "Consumption values and market choices: theory and applications," Journal of Marketing Research, vol. 29, no. 4, pp. 487-489, 1991.

[17] H. Abdollahpouri, R. Burke, and B. Mobasher, "Controlling popularity bias in learning-to-rank recommendation," in Eleventh ACM Conference on Recommender Systems, Association for Computing Machinery, New York, NY, USA, 2017.

[18] G. Guo, J. Zhang, and N. Yorke-Smith, "A novel recommendation model regularized with user trust and item ratings," IEEE Transactions on Knowledge and Data Engineering, vol. 28, no. 7, pp. 1607-1620, 2016.

[19] R. Andersen, C. Borgs, J. Chayes et al., "Trust-based recommendation systems: an axiomatic approach," in Proccedings of the International Conference on World Wide Web, pp. 199-208, Beijing, China, April 2008.
[20] R. Andersen, C. Borgs, F. Jennifer et al., "Trust-based recommendation systems: an axiomatic approach," in Proceedings of the 17th International Conference on World Wide WebWWW'08, pp. 199-208, New York, NY, USA, 2016.

[21] C. N. Ziegler and G. Lausen, "Spreading activation models for trust propagation," in Proceedings of the IEEE International Conference on E-Technology, E-Commerce and E-Service, pp. 83-97, Taipei, Taiwan, 2004.

[22] U. Kuter and J. Golbeck, "Using probabilistic confidence models for trust inference in web-based social networks," ACM Transactions on Internet Technology, vol. 10, no. 2, pp. 1-23, 2010.

[23] H. Liu, F. Xia, Z. Chen, N. Y. Asabere, J. Ma, and R. Huang, "Trucom: exploiting domain-specific trust networks for multicategory item recommendation," IEEE Systems Journal, vol. 11, no. 1, pp. 295-304, 2017.

[24] S. Asghari and K. Azadi, "A reliable path between target users and clients in social networks using an inverted ant colony optimization algorithm," Karbala International Journal of Modern Science, vol. 3, no. 3, pp. 143-152, 2017.

[25] S. Deng, L. Huang, G. Xu, X. Wu, and Z. Wu, "On deep learning for trust-aware recommendations in social networks," IEEE Transactions on Neural Networks \& Learning Systems, vol. 28, no. 5, pp. 1164-1177, 2016.

[26] M. Ghavipour and M. R. Meybodi, "Trust propagation algorithm based on learning automata for inferring local trust in online social networks," Knowledge-Based Systems, vol. 143, 2017.

[27] R. R. Mccrae and P. T. Costa Jr., "Validation of the five-factor model of personality across instruments and observers," Journal of Personality and Social Psychology, vol. 52, no. 1, pp. 81-90, 1987.

[28] A. L. Chapman, "Review of the Cambridge handbook of personality psychology," Canadian Psychology/Psychologie Canadienne, vol. 52, no. 2, pp. 141-142, 2011.

[29] S. Simon, Kaplan and Sadock's Comprehensive Textbook of Psychiatry, Vol. 1 and 2, Wolters Kluwer Health/Lippincott Williams \& Wilkins, Philadelphia, PA, USA, 7th edition, 2001.

[30] R. B. Murray, A. M. Ryan, and N. Schmitt, "Personality and work. reconsidering the role of personality in organizations," Administrative Science Quarterly, vol. 49, no. 3, pp. 472-474, 2004.

[31] X. Feng, "Study on customer personality characteristics and relationship outcomes using sem analysis," Applied Mechanics and Materials, vol. 462-463, pp. 841-844, 2013.

[32] B. Caci, M. Cardaci, M. E. Tabacchi, and F. Scrima, "Personality variables as predictors of facebook usage," Psychological Reports, vol. 114, no. 2, pp. 528-539, 2014.

[33] M.-G. Cojocaru, H. Thille, E. Thommes, D. Nelson, and S. Greenhalgh, "Social influence and dynamic demand for new products," Environmental Modelling \& Software, vol. 50, no. 12, pp. 169-185, 2013.

[34] Y. A. Hamburger and E. Ben-Artzi, "The relationship between extraversion and neuroticism and the different uses of the internet," Computers in Human Behavior, vol. 16, no. 4, pp. 441-449, 2000.

[35] J. Tang, H. Gao, H. Liu, and A. Das Sarma, "etrust:understanding trust evolution in an online world," in Proceeding of the ACM SIGKDD International Conference on Knowledge Discovery and Data Mining, vol. 253-261, Beijing, China, August 2012.

[36] J. Tang, H. Gao, A. D. Sarma, Y. Bi, and H. Liu, "Trust evolution: modeling and its applications," IEEE Transactions 
on Knowledge and Data Engineering, vol. 27, no. 6, pp. 1724-1738, 2015.

[37] M. Jamali and M. Ester, "Trustwalker:a random walk model for combining trust-based and item-based recommendation," in Proceedings of the ACM SIGKDD International Conference on Knowledge Discovery and Data Mining, pp. 397-406, Washington, DC, USA, July 2009.

[38] W. Jiang, G. Wang, and J. Wu, "Generating trusted graphs for trust evaluation in online social networks," Future Generation Computer Systems, vol. 31, no. 1, pp. 48-58, 2014. 